\title{
Structured transparent low emissivity coatings with high microwave transmission
}

\author{
Olivia Bouvard ${ }^{1}$ (D) Matteo Lanini ${ }^{2} \cdot$ Luc Burnier $^{1} \cdot$ Reiner Witte $^{3} \cdot$ \\ Bernard Cuttat $^{4} \cdot$ Andrea Salvadè $^{2} \cdot$ Andreas Schüler $^{1}$
}

Received: 6 June 2016/ Accepted: 14 December 2016

(C) Springer-Verlag Berlin Heidelberg 2016

\begin{abstract}
In order to reduce the energy consumption of buildings, modern windows include metal-containing coatings. These coatings strongly attenuate the microwaves used for mobile communications. Here, we present a novel approach to improve radio signal transmission by structuring a low emissivity coating. Laser ablation is used to scribe a line pattern on the coating. The microwave attenuation of the initial coating ranges between -25 and $-30 \mathrm{~dB}$ between $850 \mathrm{MHz}$ and $3 \mathrm{GHz}$. The optimized patterning reduces it down to $-1.2 \pm 0.6 \mathrm{~dB}$. The fraction of the ablated area is relatively low. Our experimental results show that it is possible to reach a level of attenuation close to that of a glass substrate by removing less than $4 \%$ of the coating area. The ablated lines are thin enough to not be noticed in most common lighting situations. Therefore, we achieve a dual spectral selectivity: the coated glass is transparent in the visible range, reflective in the infrared and nearly as transparent as its glass substrate to microwaves. Additionally, numerical simulations were performed and show that the attenuation at grazing incidences is dominated by the behaviour of the glass substrate.
\end{abstract}

Olivia Bouvard

olivia.bouvard@epfl.ch

1 Solar Energy and Building Physics Laboratory, Ecole Polytechnique Fédérale de Lausanne (EPFL LESO-PB), Station 18, 1015 Lausanne, Switzerland

2 Telecom Telemetry High Frequency Lab, SUPSI, Dipartimento Tecnologie Innovative, University of Applied Sciences of Southern Switzerland, Galleria 2, Manno, Switzerland

3 Class 4 Laser Professionals AG, Bahnhofstrasse 43, 3400 Burgdorf, Switzerland

4 AGC Verres Industriels SA, Quartier Verreries 25, 2740 Moutier, Switzerland
To the best of our knowledge, it is the first time that experimental evidence for the combination of such properties is reported and that detailed experimental data are compared to numerical simulations. We anticipate that our findings will be of major importance for the building and transportation sectors.

\section{Introduction}

Energy-saving windows contain low emissivity (low-e) coatings. These coatings usually consist of a stack of dielectric and metallic thin films, generally $1-3$ layers of silver along with $8-15$ dielectric layers. The envelope of trains is usually made of metal. If such train is equipped with windows containing metallic coatings, the electromagnetic waves used for telecommunications are strongly attenuated as in a Faraday cage. This problem was also identified in buildings equipped with modern windows [1-3]. When the attenuation is too strong, repeaters are used to amplify the signal. These need to be replaced whenever communication standards change and their cost is non-negligible. The currently used communication standards include GSM (Global System for Mobile communications, $880-960 \mathrm{MHz}$ ) and UMTS (Universal Mobile Telecommunications System, 1920-2170 MHz). Therefore, there is a need for a material engineered so that it could reflect the infrared and transmit the microwaves while conserving transparency in the visible: a low emissivity coating with high microwave transmission.

Properties not found in nature can be obtained by metamaterials. They are usually composed of structures which are much smaller than the wavelength of the electromagnetic waves with which it interacts. Metamaterials have attracted interest worldwide because they allow to 
achieve new properties such as a negative refractive index [4-6] or exceptional mechanical properties $[7,8]$.

Frequency selective surfaces (FSS) are usually a twodimensional periodic array of patch or aperture elements with dimensions in the order of magnitude of the wavelength or smaller [9, 10]. Previous studies [11-14] investigated complex-shaped FSS specifically designed to transmit narrow frequency bands (e.g. GSM 880-960 MHz). This narrow transmission band makes the structure dependent of changes in communication technologies. Furthermore, the surface of removed coating is rather large (12.35\% for the Ullah et al. design [14]). As the effect on the radiative thermal properties is rather proportional to the surface of ablated coating, the ablated area should be kept as small as possible.

The aim of this work is to achieve a combination of three main properties: transparency to microwaves for telecommunications, negligible losses in the thermal performances of the window and undisturbed visibility through the window. To reach these goals, we used laser ablation to prepare structured low emissivity coatings. We characterized these samples by SEM, confocal laser scanning optical microscopy, visible and infrared spectrophotometry, in terms of visual appearance of the pattern and by the spectral measurement of microwave transmission. The microwave transmission was also simulated, and results were compared to experimental data.

\section{Theory}

A patterned conductive coating can be described as a FSS. Various techniques exist for analysing frequency selective surfaces [15]. The analytical averaged approach presented by Costa et al. [16] can be used in order to analyse the frequency response of the FSS. A square patch pattern can be considered as a non-resonant structure with capacitive behaviour. The system can be modelled using an electrical circuit equivalence, where a resistor $\left(R_{\text {coating }}\right)$ simulates a conventional low-e coating (see Fig. 1 a), and a capacitor $\left(C_{\mathrm{fss}}\right)$ in series with a resistor $\left(R_{\mathrm{fss}}\right)$ represents a coating patterned in square patches (see Fig. $1 \mathrm{~b}$ ). $Z_{0}$ represents the impedance of air and $Z_{0, \text { in }}=Z_{0, \text { out }}=377 \Omega$. The pattern can be described by the geometrical parameters linewidth w and periodicity D (see Fig. 1c).

For the considered electrical circuit with a resistor and a capacitor in series, the cut-off frequency $f_{-3 \mathrm{~dB}}$ corresponding to an attenuation of $3 \mathrm{~dB}$ is given by:

$f_{-3 \mathrm{~dB}}=\frac{1}{2 \pi \cdot\left(R_{\mathrm{fss}}+\frac{Z_{0}}{2}\right) \cdot C_{\mathrm{fss}}}$

In the case of a capacitive square patch and at normal incidence, $C_{\mathrm{fss}}$ is calculated as follows [16]:

$C_{f s s}=\frac{D \varepsilon_{0}\left(\varepsilon_{r 1}+\varepsilon_{r 2}\right)}{\pi} \ln \left(\frac{1}{\sin \left(\frac{\pi w}{2 D}\right)}\right)$

where $D$ is the periodicity of the FSS; $w$ is the linewidth between the conductive patches; $\varepsilon_{0}$ is the vacuum permittivity; and $\varepsilon_{r 1}, \varepsilon_{r 2}$ are the relative dielectric permittivities of the surrounding mediums (i.e. air and glass).

If the ratio $w / D$ is maintained constant, $C_{\mathrm{fss}}$ becomes independent of $w$ and is proportional to $D$.

The surface resistance $R_{\mathrm{fss}}$ is given by [17]:

$R_{\mathrm{fss}} \approx R_{\mathrm{coating}} \frac{D^{2}}{(D-w)^{2}}$

where $R_{\text {coating }}$ is the sheet resistance of the patches of the conductive coating. Therefore, if the width $w$ is very small as compared to the distance D, the surface resistance $R_{\mathrm{ffs}}$ will be similar to the resistance of the patches of the conductive coating $R_{\text {coating }}$.

for $w<<D: R_{\mathrm{fss}} \approx R_{\text {coating }}$

For patterned conductive low emissivity coatings on glass substrates, and for frequencies clearly below the plasma frequency of the free charge carriers, the following properties are typical [18]: $\varepsilon_{\mathrm{r}}$ glass $=7.0$, coating sheet resistance: $4.0 \Omega / \mathrm{sq}$. Using these values, the cut-off frequency $f_{-3 \mathrm{~dB}}$ can be calculated as a function of $w$ and $D$.

Figure 2a shows the behaviour of $f_{-3 \mathrm{~dB}}$ when w is kept constant at a value of $35 \mu \mathrm{m}$ and $D$ is varied from 0.5 to
Fig. 1 a Equivalent circuit of an electrically conductive coating; $\mathbf{b}$ equivalent circuit of a capacitive square patch pattern with resistive elements; c geometrical parameters of a square patch pattern (a)

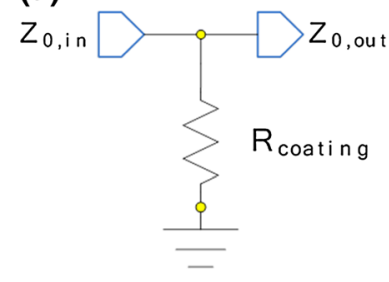

(b)

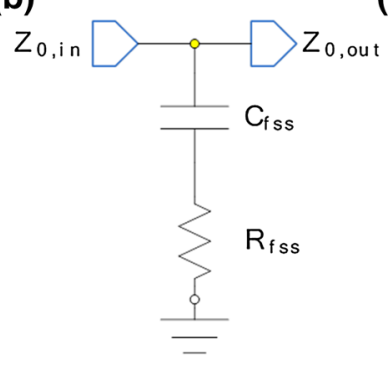

(c)

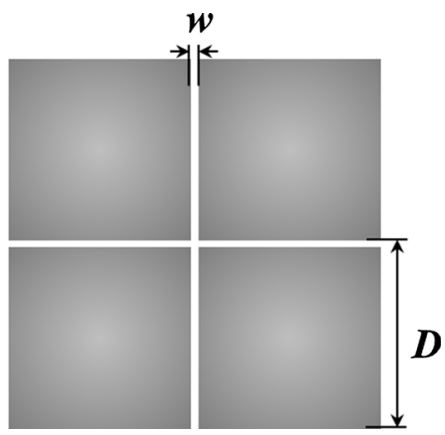


Fig. 2 a Cut-off frequency $f_{-3 \mathrm{~dB}}$ as a function of $D$ for $w=35 \mu \mathrm{m}, \mathbf{b}$ cut-off frequency

$f_{-3 \mathrm{~dB}}$ as a function of $\mathrm{w}$ for $D=4 \mathrm{~mm}$
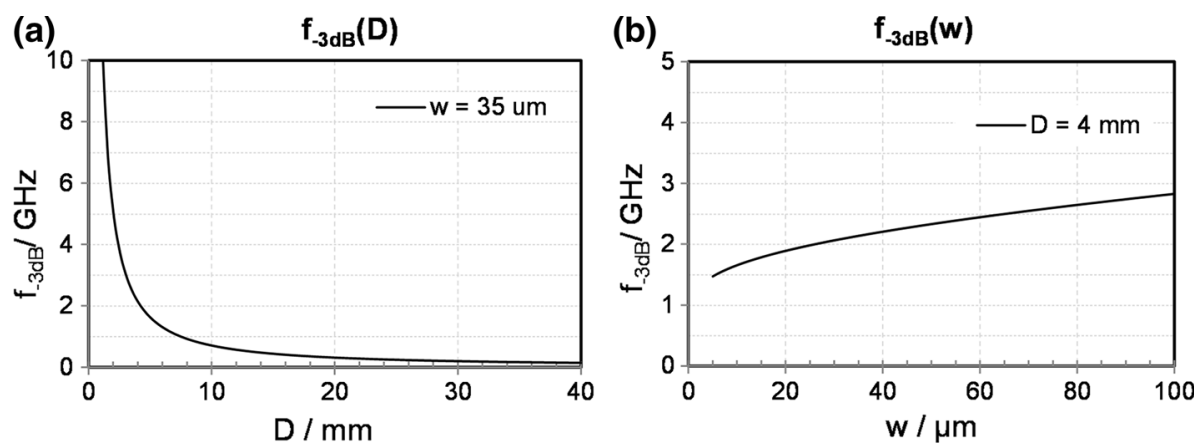

$40 \mathrm{~mm}$. For decreasing $\mathrm{D}, f_{-3 d B}$ is monotonously increasing. At $D=2 \mathrm{~mm}$, a cut-off frequency of $5.1 \mathrm{GHz}$ is obtained.

Figure $2 \mathrm{~b}$ shows the behaviour of $f_{-3 \mathrm{~dB}}$ when $D$ is kept constant at a value of $2 \mathrm{~mm}$, and $\mathrm{w}$ is varied from 1 to $100 \mu \mathrm{m}$. For small $w, f_{-3 \mathrm{~dB}}$ goes approximately with $\ln (D /$ w) (compared with Eq. 2). For increasing w, $f_{-3 \mathrm{~dB}}$ is monotonously increasing, with decreasing slope. For $w \geq 60 \mu \mathrm{m}$, cut-off frequencies $f_{-3 \mathrm{~dB}}$ above $2.5 \mathrm{GHz}$ are obtained.

\section{Methods}

\subsection{Laser scribing of low emissivity coating}

Commercial glass for energy-saving windows from AGC Verres Industriels Moutiers was processed. Glass samples with a thickness of $4 \mathrm{~mm}$ and a size of $500 \mathrm{~mm} \times 500 \mathrm{~mm}$ were used. They were coated with a "Low-e Top $\mathrm{N}+\mathrm{T}$ " low emissivity coating. After laser scribing, the glass panes were assembled into double glazing. They are composed of a first glass pane of grey glass $4 \mathrm{~mm}$ thick, a 12-mm air gap and the coated glass pane. With these dimensions, the attenuation of microwaves can be measured.

Laser ablation of the coating was performed using a 1064-nm nanosecond fibre laser. Patterns (lines or grid) were drawn using a human interface device defining the movement of the laser head, the sample staying motionless. Lines were ablated by pulses of laser at a frequency of $10 \mathrm{kHz}$ with a speed of $6 \mathrm{~m} / \mathrm{min}$ and a power of $10.5 \mathrm{~W}$. Samples were processed with the laser beam incident from the backside for better precision [19]. Focus and power were adjusted in order to obtain an ablation width appropriate for interrupting the electrical conductivity. The latter being measured using a high voltage multimeter. Different laser parameters have been tested in order to obtain the thinnest line while still providing electrical insulation.

\subsection{Characterization of the coating and of the ablated area}

A Keyence confocal laser scanning optical microscope was used to determine the width of the ablated line and to check the regularity of the line on the day of processing. Further investigations by scanning electron microscope (SEM) were conducted using a FEI XLF30-FEG for secondary electron imaging at $5 \mathrm{kV}$ (Everhart-Thornley secondary electron detector and Schottky FEG electron gun). Optical transmittance of the coated, uncoated and structured coating was measured, using a Zeiss diode array spectrometer (MCS 601 and MCS 611), in the ultraviolet (UV), visible and near infrared (NIR) range. A FTIR spectrometer (SE 900 Sentech) was used for investigating the optical properties of thin films in the middle infrared range, from 2.5 to $16.7 \mu \mathrm{m}$. The spectral reflectance of a coated glass sample was measured before and after engraving. Applying Kirchoff's law, which states that the emissivity, $\varepsilon$, of a body equals its absorptance at thermal equilibrium, the emissivity can be obtained by subtracting the reflectance from 1. The reflectance $\rho$ is the integral of the spectral reflectance weighted by the emission of a black body at $283 \mathrm{~K}$. The influence of the alteration of emissivity on the thermal performances of the window was evaluated by calculating the $U$ value or thermal transmittance of the centre of the glazing, $U$, according to the standard EN 673 [20] (Eq. 5, 6):

$\frac{1}{U}=\frac{1}{h_{e}}+\frac{1}{h_{\mathrm{t}}}+\frac{1}{h_{i}}$

$h_{e}$ and $h_{i}$ are the external and internal heat transfer coefficient. $h_{t}$ is the total conductance of the glazing. The modification of the emissivity of the coated glass $\varepsilon_{2}$ will impact the radiation conductance $h_{r}$ which is a contribution to $h_{t}$. All other parameters were kept constant and as specified in EN 673.

$h_{\mathrm{r}}=\left(\frac{1}{\varepsilon_{1}}+\frac{1}{\varepsilon_{2}}-1\right)^{-1} \cdot 4 \cdot \sigma \cdot T^{3}$ 


\subsection{Measurement and numerical simulation of the microwaves transmission}

In the scientific literature, different approaches are presented to determine the microwave attenuation or radio frequency (RF) insertion loss of a material [21]. In our work, we adopted a free-space approach (see Fig. 3a, b) that is recognized to be a good trade-off in of terms of measurement accuracy in a dynamic range of $50-60 \mathrm{~dB}$, repeatability, simplicity of the test fixture and fast measurement time [22, 23].

The window is composed of several interfaces (double glazing) separated by a metallic spacer; the construction tolerances are in the order of $\pm 1 \mathrm{~mm}$. The measured prototype dimensions are $50 \mathrm{~cm} \times 50 \mathrm{~cm}$. To achieve the necessary accuracy, the set-up includes a large metallic shield to minimize the edge effect at the panel borders (see Fig. 3c, d) and the measurement is performed with a 2-port Vector Network Analyzer (VNA) with time gating capability [24]. The set-up is composed of: (a) A sample holder with an additional metallic shield and EMI gaskets for samples under test (SUT) smaller than $60 \mathrm{~cm} \times 60 \mathrm{~cm}$.

(b) A Vector Network Analyzer Agilent PNA E8364B $10 \mathrm{MHz}$ to $50 \mathrm{GHz}$, with time domain (Option 010);

(c) $2 \times \mathrm{R} \& \mathrm{~S}^{\circledR} \mathrm{HL} 050$ Antenna, High Gain Log-Periodic Antenna $850 \mathrm{MHz}$ to $26.5 \mathrm{GHz}$;

(d) $4 \times$ Emerson \& Cuming, ECCOSORB ${ }^{\circledR}$ AN 79 broadband absorbers;

(e) $2 \times 2 \mathrm{~m}$ High-performance microwave coaxial cables, Huber + Suhner, Sucoflex ${ }^{\circledR} 100$;

The time-gated measurement results allow the cancellation of multipath effects of the room (walls, roof floor) but not of the edge effect (scattering on SUT edge). In fact, we observed some fading (Rician like) on the envelope of the measured curve. This effect is present only on samples less than $100 \mathrm{~cm}$ by $100 \mathrm{~cm}$ as the longest path cannot be discriminated in the time domain and the metallic screen introduces standing waves. Standing waves can be (a)
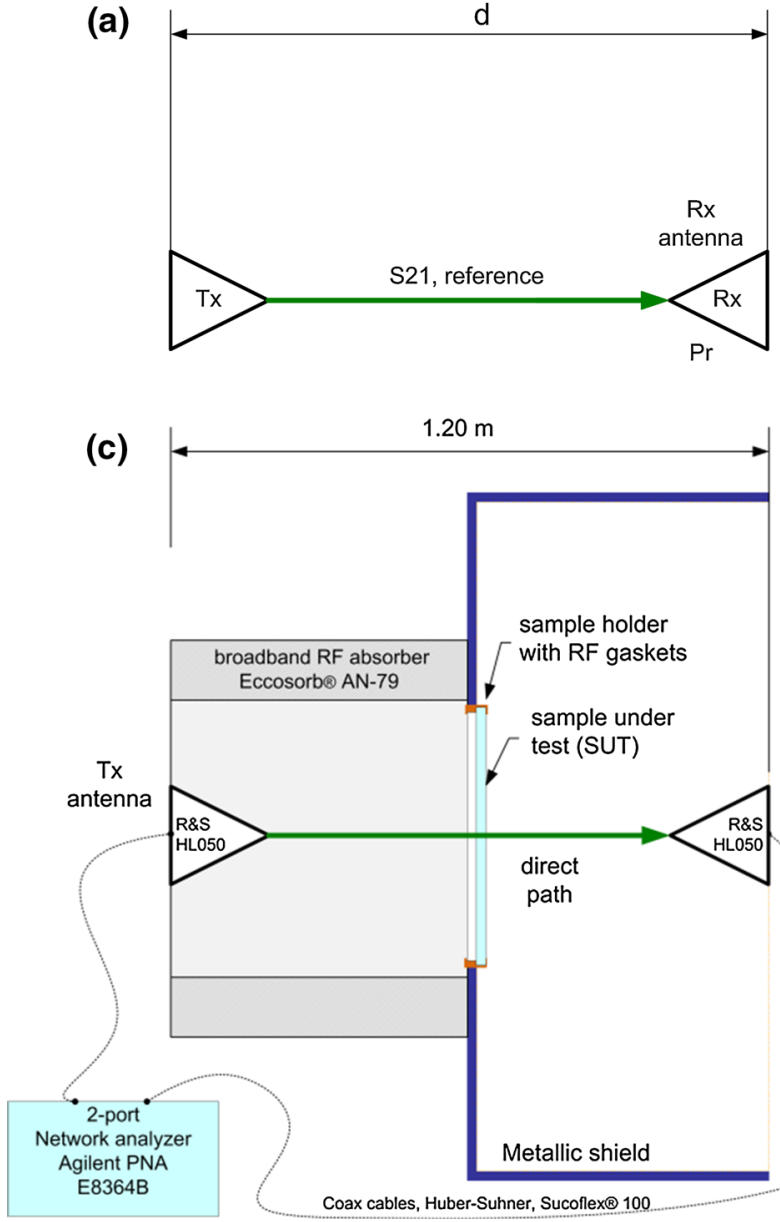

Fig. 3 Free-space method consists of a a reference measurement of the free-space loss and $\mathbf{b}$ the measurement with the SUT. $\mathbf{c}$ To deal with reflections/diffractions, the experimental set-up was modified (b)

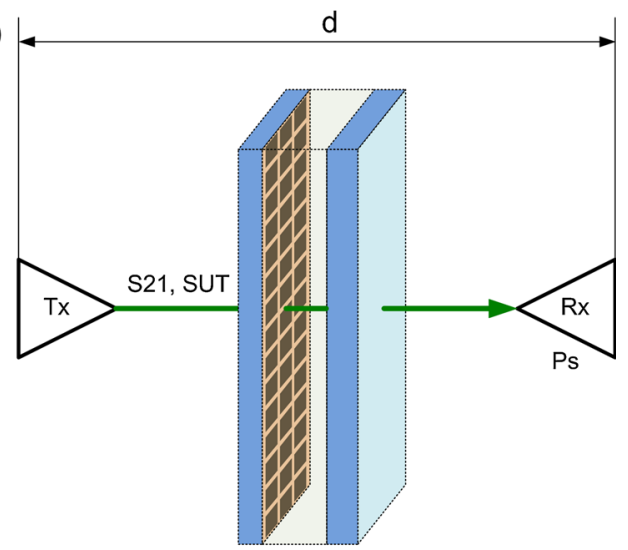

(d)

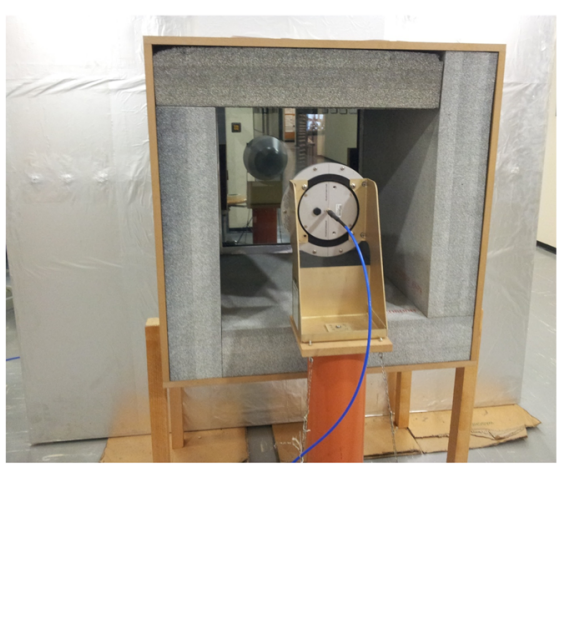

with a metallic shield and a time-gated vector network analyzer (VNA). d Picture of the test fixture and a glazing (SUT) 
measured by averaging several measurements taken in different positions [25] or by averaging the results in the frequency domain. In the present work, the antennas were fixed in one position (to keep a constant zero reference); thus, we applied a post-treatment using a cubic smoothing spline to minimize the ripple on the SE curves [26].

The structured coating was simulated with the 2.5D EM simulator Ansys ${ }^{\circledR}$ Designer $^{\mathrm{TM}}$ (version 15.0.0), a commercial tool that allows the analysis of infinite structures acting as frequency selective surfaces (FSS) with fast and accurate simulations over an extremely wide bandwidth. The solver is based on the solution of mixed potential equations (multilayered media Green's functions) by means of the method of moments [27, 28]. The following properties were used [18]: $\varepsilon_{\mathrm{r}}$ glass $=7.0$, coating sheet resistance: $4.0 \Omega / \mathrm{sq}$.

\section{Results}

\subsection{Characterization of the ablated area}

The ablated area is kept small thanks to very narrow lines. A nanosecond fibre laser (1064 nm) was used to scribe fine lines on a low emissivity (low-e) coating. The frequency of the pulse was $10 \mathrm{kHz}$, and the displacement speed was $6 \mathrm{~m} / \mathrm{min}$.

Figure 4 displays images that were obtained by confocal laser microscopy (a, b) and scanning electron microscopy (c, d). Figure $4 \mathrm{a}$ shows the detail of an ablated line; the dark area corresponds to the low-e coating. Due to the use of a nanosecond pulsed laser, the lines consist of a succession of overlapping discs corresponding to the focal spot of the laser. The overlap is in the order of $70-75 \%$ of the disc surface. The width of the resulting line is approximately 40 microns. Figure $4 \mathrm{c}$ presents an enlarged view. On the edge of the ablated line, an accumulation of material is observed. The material of the low-e coating is ejected to the side of the laser beam. After ablation, it was verified that the electrical conductivity between two adjacent zones was effectively interrupted using an isolation tester for a representative number of zones. The width of 35-40 microns was selected because it proves sufficient to electrically insulate two adjacent zones. This width could be reduced further in dust-free conditions. The laser ablation was then performed in parallel lines on the whole sample surface. Subsequently, an array of lines perpendicular to the first ones was ablated. The resulting intersection is shown in Fig. 4b, d. It can be observed that there is no material deposition on the lines around the intersection point. Therefore, there are no short circuits in these areas and square patches of conductive coatings are formed. The interspacing between lines was varied between 2 and $40 \mathrm{~mm}$. For a linewidth of 40 microns and a spacing of $40 \mathrm{~mm}$, the corresponding ablated area is only $0.2 \%$. For the same linewidth and a spacing of $2 \mathrm{~mm}$, the ablated area represents $4 \%$ of the coating.

The ablated area should be kept as small as possible to preserve the low emissivity and therefore the insulating performances of the window. The reflectance in the midinfrared region $(2.5-16.7 \mu \mathrm{m})$ measured using an FTIR spectrophotometer is displayed in Fig. 5. The solid line in Fig. 5 represents the conventional coating, and its reflectance varies from $94 \%$ at $3 \mu \mathrm{m}$ to $97 \%$ between 10 and $16 \mu \mathrm{m}$. The dashed line shows the values measured after the laser engraving. The reflectance is slightly lower with values ranging from $91 \%$ at $3 \mu \mathrm{m}$ to $95 \%$ around $9 \mu \mathrm{m}$. The feature observable around $4.4 \mu \mathrm{m}$ is due to the absorption of water and was not taken into consideration for the calculation of emissivity.

Table 1 displays the emissivity determined from the FTIR measurement and the corresponding thermal transmittance, or U value, calculated according to Eqs. 5, 6 [20]. For this calculation, a double glazing composed of two glass panes (one grey uncoated glass, $d_{1}=5 \mathrm{~mm}$ and one coated clear glass, $d_{2}=4 \mathrm{~mm}$ ), an air gap of $s=12 \mathrm{~mm}$ and a mean temperature of $T=283 \mathrm{~K}$ were used.
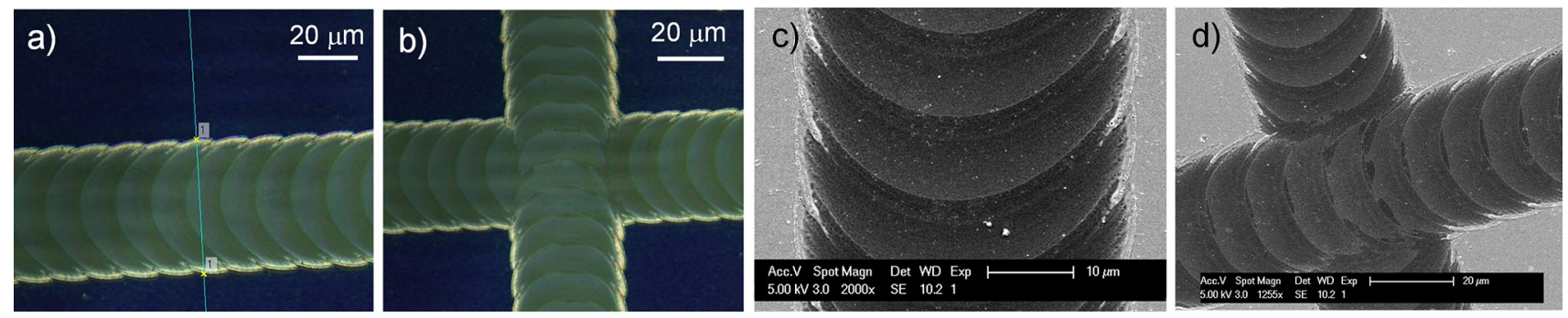

Fig. 4 a Image of a line ablated by laser pulses with confocal laser microscopy. The dark area represents the coating. On the light part, the superimposition of the pulses can be observed. b Image of an intersection by confocal laser microscopy. c Observation by scanning electron microscopy (SEM) of the linewidth. On the side of the

ablated area, some accumulation of material can be seen. $\mathbf{d}$ Details of the intersection by SEM. The width of the ablated line depends on the laser power and focus. In this study, it was kept between 35 and 40 microns 


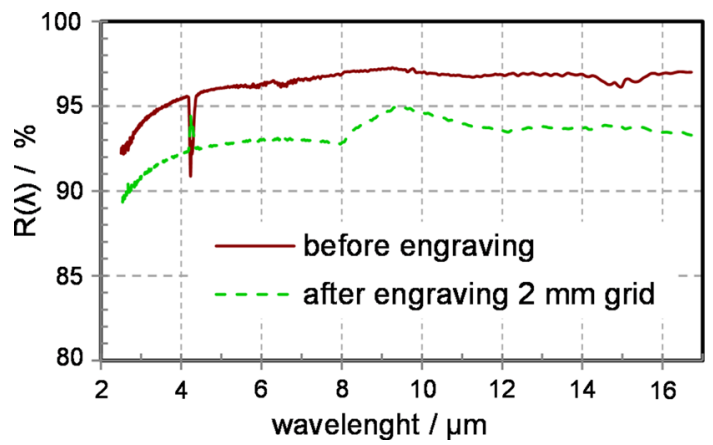

Fig. 5 Spectral reflectance of the coated glass in the mid-infrared region before and after laser engraving (ablated lines width of $w=37 \pm 2 \mu \mathrm{m}$, spacing $D=2 \mathrm{~mm}$ in grid pattern). The feature at approximately $4 \mu \mathrm{m}$ is due to the absorption of water

Table 1 Results of emissivity determined form the FTIR measurements performed before and after engraving on the same coated glass

\begin{tabular}{lll}
\hline & $\varepsilon_{\text {exp. }} / \%$ & $U_{\text {calc. }} / \mathrm{W} \mathrm{m}^{-2} \mathrm{~K}^{-1}$ \\
\hline Before engraving & 3.3 & 1.6 \\
After engraving & 6.5 & 1.7
\end{tabular}

The $U$ value was calculated taking into account the measured emissivity for the full low-e coating and the engraved one

The laser treatment increases the emissivity of the coating of $3.2 \%$. It corresponds to an increase of $0.1 \mathrm{~W} \mathrm{~m}^{-2} \mathrm{~K}^{-1}$ for the calculated $\mathrm{U}$ value, which is rather small. Hence, we can expect a minor difference between the conventional glazing and the one with a structured glazing in terms of overall thermal performances.

\subsection{Optical properties and visual appearance}

After engraving, the glass panes were assembled into double glazing to resemble a common window. Optical measurements were performed on the obtained samples. Figure 6 displays the direct transmittance in the solar spectral range (UV, visible, NIR) of the double glazing composed of the conventional low-e coating and the one with a structured coating ( $2 \mathrm{~mm}$ spacing grid pattern). The two spectra are similar; the three peaks visible around 400,600 and $800 \mathrm{~nm}$ are due to the grey glass used as second glass pane. A difference can be observed from 1200 to $2100 \mathrm{~nm}$ where the structured coating is slightly more transparent. This can be explained since a part of the low-e coating which also acts as a solar protection is removed. The solar protection aims at reducing the amount of invisible solar energy (mainly NIR) entering the room to prevent overheating. From 400 to $800 \mathrm{~nm}$, the measured values are comparable. It shows that the laser treatment does not alter the direct transmittance in the visible range.

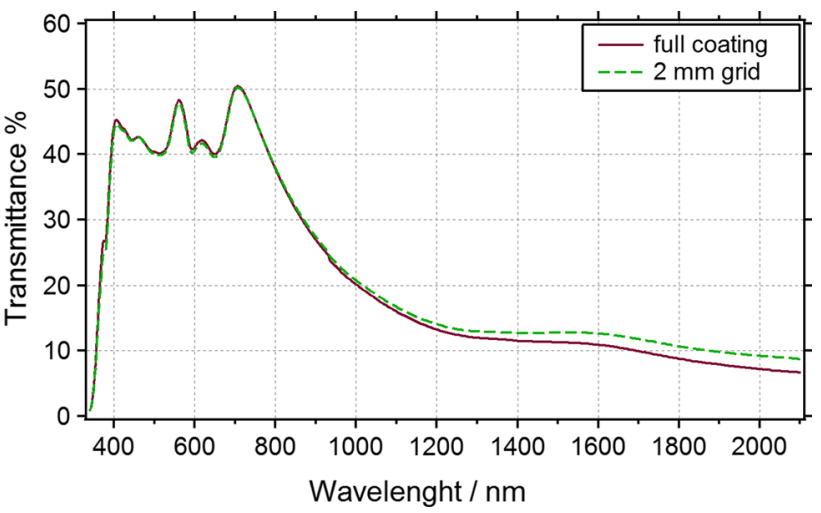

Fig. 6 Optical transmittance in the solar spectrum range of the double glazing with full low-e coating (full coating) and with laserstructured low-e coating (ablated lines width of $w=37 \pm 2 \mu \mathrm{m}$, spacing $D=2 \mathrm{~mm}$ in grid pattern)

As a result, the pattern is not visible in most lighting situations. Figure 7 displays photographs of the sample with a 2-mm line spacing. In Fig. 7a, representing a common situation, the pattern is not visible even though the focus was on the window. Figure $7 \mathrm{~b}$ exhibits the specific case with strong backlighting. The pattern appears in the reflection of the sun beam. The other parts of the window remain clear. In Fig. $7 \mathrm{c}$, d, a dark environment was set up in the laboratory. For the image in Fig. 7c, a strong illumination was directed towards the sample at a grazing incidence to reveal the pattern, while in Fig. $7 \mathrm{~d}$, in ambient light, the pattern is not visible. Along with a good transmission of microwaves, the low visibility of this laser pattern is a key element for user acceptance.

\subsection{Measured and simulated attenuation of the microwaves}

The interruption of the electrical conductivity at a scale much smaller than the wavelength considered is expected to enhance microwave transmission. As presented previously, the size of the pattern (D) modifies the capacitive behaviour of the coating. Figure 8a illustrates the measured microwave attenuation of the studied double glazing, composed of a first glass pane of grey glass with a thickness of $5 \mathrm{~mm}$, a 12-mm air gap, and a second glass pane, $4 \mathrm{~mm}$ thick, either coated with a commercial low-e coating, uncoated (float glass) or with a laser-structured coating. The pattern obtained by laser ablation is composed of parallel and perpendicular straight lines forming a grid, thus resulting in square patches of low-e coating as described in Fig. 1c. The spacing D between the lines in horizontal and vertical directions is identical and is varied from 2 to $40 \mathrm{~mm}$.

The window with a full low-e coating exhibits a strong attenuation of the microwaves $(-25$ to $-32 \mathrm{~dB})$ in the 

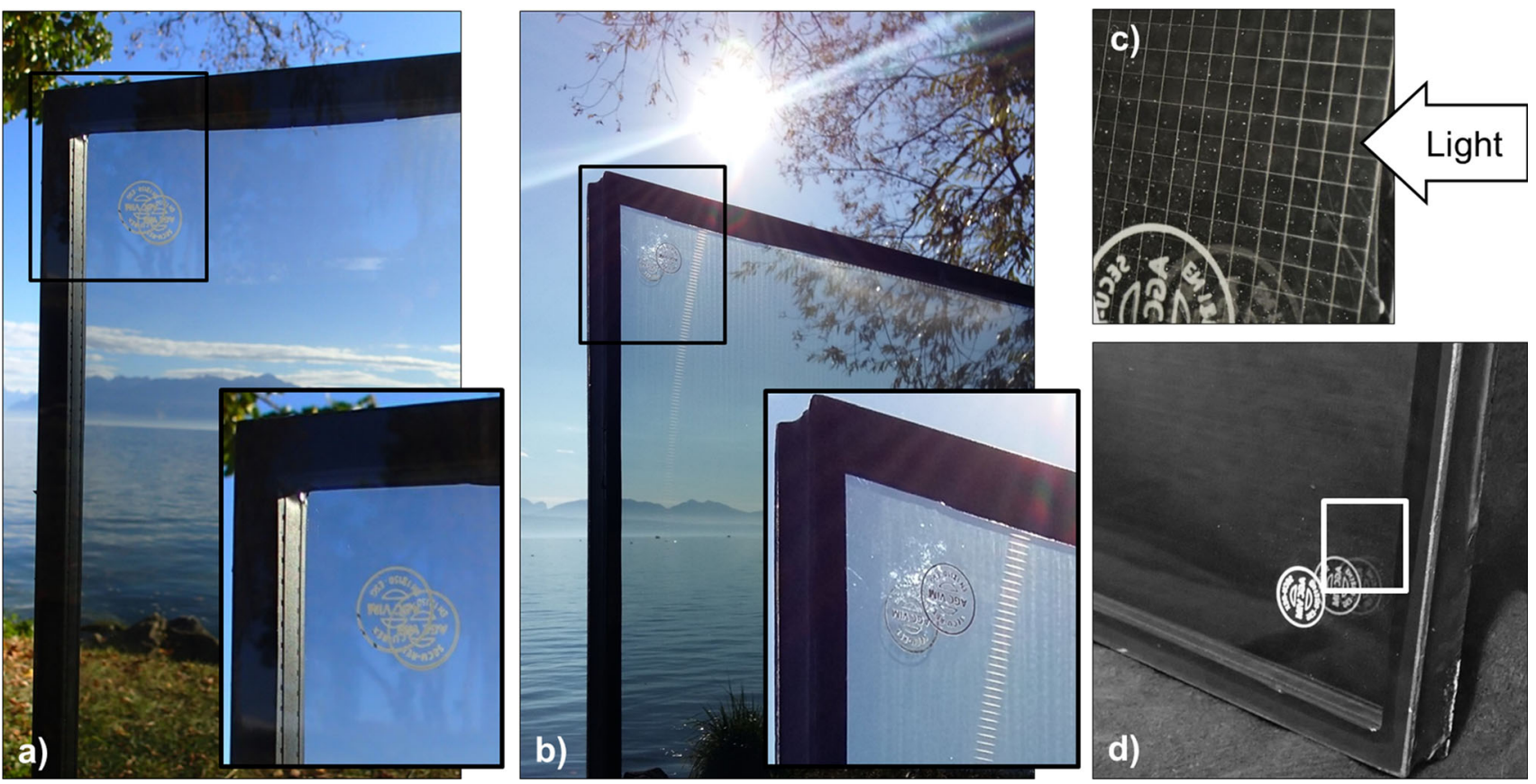

Fig. 7 Photograph of a double glazing window with laser-structured low-e coating (pattern with a line spacing of $2 \mathrm{~mm}$ ). a Photograph of the sample in common outdoor conditions. The focus is on the frame, the background is blurry, but the pattern is not discernible. A zoom on the top left corner of the glass is presented in the inset. $\mathbf{b}$ Contre-jour photograph of the sample; in these backlit conditions the pattern can

be distinguished in reflection. The inset shows a zoom of this reflection. $\mathbf{c}$ The pattern is revealed in extreme conditions, here by directing a strong illumination on the sample at grazing incidence. d Photograph of the sample in indoor conditions with artificial light and a dark background

Fig. 8 a) Measured attenuation in the mobile communication range $(0.850-5 \mathrm{GHz})$ for the double glazing without coating (float glass), with full low-e coating (full coating) and with laser-structured low-e coatings (ablated lines width of $w=37 \pm 2 \mu \mathrm{m}$, spacing $D$ from 2 to $40 \mathrm{~mm}$ in grid pattern, respectively, $4-0.2 \%$ of ablated area). b Measured and simulated attenuation at $900 \mathrm{MHz}$ and $\mathbf{c} 2100 \mathrm{MHz}$ of double glazing as a function of the surface of coating ablated. The straight lines represent the float glass without coating assembled as double glazing
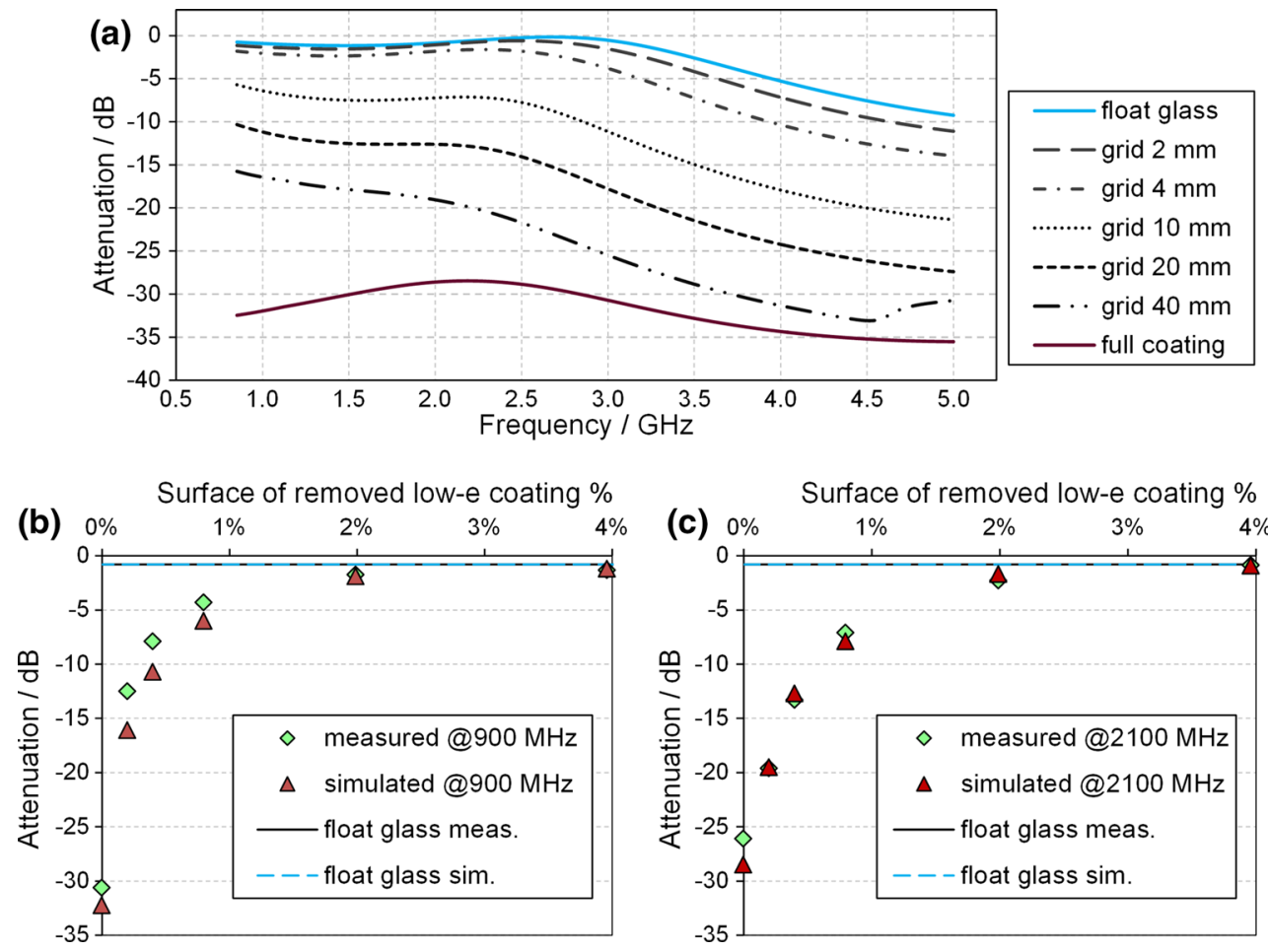
range of interest $(850 \mathrm{MHz}-3 \mathrm{GHz})$. In contrast, the attenuation of microwaves is very low for a double glazing composed of uncoated glass, less than $-1.3 \mathrm{~dB}$ for frequencies below $3 \mathrm{GHz}$. For the laser-structured coatings, the microwave attenuation varies dramatically with the size of the pattern D. Nevertheless, the pattern with $D=40 \mathrm{~mm}$ which represents a surface of ablated coating as small as $0.2 \%$ already reduces the attenuation from -30 to $-12 \mathrm{~dB}$ at $850 \mathrm{MHz}$. As the line spacing is decreased, the microwave attenuation is decreased. For the pattern with $D=4 \mathrm{~mm}$, the attenuation is no stronger than $5 \mathrm{~dB}$ between $850 \mathrm{MHz}$ and $3 \mathrm{GHz}$. The values obtained for the smallest spacing studied $(D=2 \mathrm{~mm})$ are extremely close to the one of the substrates. The difference between the two samples is ranging from 0.2 to $0.6 \mathrm{~dB}$ below $3 \mathrm{GHz}$. By removing less than $4 \%$ of the coating, a transparency to microwaves close to that of uncoated glass is achieved.

In order to compare the experiments to the theoretical expectations, simulations were performed using the ANSYS $^{\circledR}$ Designer $^{\mathrm{TM}}$ software. The comparison between experimental and simulated results is presented in Fig. $8 \mathrm{~b}$ at $900 \mathrm{MHz}$ and Fig. 8c at $2100 \mathrm{MHz}$.

The attenuation is shown as a function of the surface of low-e coating that is removed. A line spacing of $D=4 \mathrm{~mm}$ represents an ablated area of $2 \%$ considering a linewidth of $w=40$ microns. The values at $0 \%$ of removed coating represent the conventional low-e coating. At $900 \mathrm{MHz}$, both measured and simulated values are at $-32.2 \mathrm{~dB}$. There is a slight difference at $2100 \mathrm{MHz}$; the simulated value is $-28.5 \mathrm{~dB}$ while the measured value is $-26.1 \mathrm{~dB}$. The straight lines indicate the value for the uncoated glass; identical values were obtained for simulated and measured results, namely $-0.8 \mathrm{~dB}$ at $900 \mathrm{MHz}$ and $-0.9 \mathrm{~dB}$ at $2100 \mathrm{MHz}$.

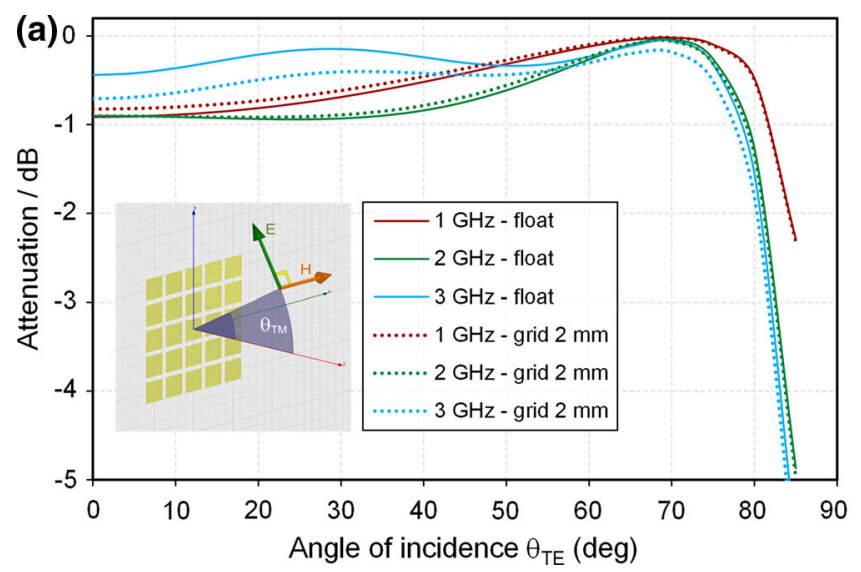

Fig. 9 Simulated attenuation from normal to near-grazing angles of incidence simulated in $\mathbf{a}$ TM and $\mathbf{b}$ TE polarization at 1,2 and $3 \mathrm{GHz}$. The straight lines represent the float glass without coating assembled
The performed simulation shows a good agreement with the experimental results. In Fig. 8b, c, we can clearly observe the impact of the size of the pattern $\mathrm{D}$ and thus the surface of ablated coating.

\section{Discussion}

It is important that the microwave attenuation remains low for a wide range of angles of incidence. Numerical simulations were performed in order to forecast the behaviour of the double glazing window with and without the structured coating. Figure 9 displays the obtained results for transverse magnetic (TM) and transverse electric (TE) polarization. The continuous lines represent the double glazing without coating while the dotted lines refer to the structured coating. The different tints (red, green, blue) refer to the considered frequencies (respectively, 1, 2 and $3 \mathrm{GHz}$ ). It can be observed that the angular-dependent response is dominated by the behaviour of the uncoated glass. The attenuation of both the uncoated glass pane and the glass with the laser-structured coating is extremely low between $850 \mathrm{MHz}$ and $3 \mathrm{GHz}$. In addition, the angular response is quite similar for 1, 2 and $3 \mathrm{GHz}$. Therefore, the proposed window with structured coating is expected to perform nearly like an uncoated glazing for a large range of frequencies and even at high angles of incidence.

A wide band pass is desired for compatibility with the future evolution of frequencies used for telecommunications. Furthermore, the angular-dependent transmittance is less critical for a wide band pass. The used grid pattern fulfils these conditions.

The cut-off frequency $f_{-3 \mathrm{~dB}}$ shall be high enough for today's and future mobile communication. This can be assured by making D sufficiently small. An upper limit for

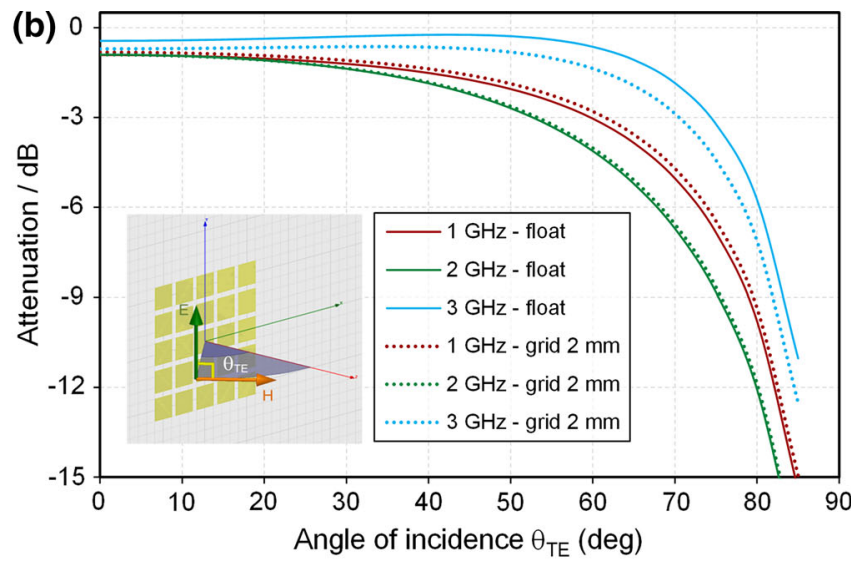

as double glazing while the dotted lines refer to the new glass with the structured low-e coating (grid $2 \mathrm{~mm}$ ) 
w can then be defined taking into account the desired ratio w/D. Too small w can result in a decrease of $f_{-3 d B}$, which is not desired. When w becomes comparable to the thickness of the conductive coating, an additional capacitive effect can be expected since the structure can then no longer be considered as two-dimensional.

Furthermore, the requirement of a low thermal emissivity implies that the surface of ablated coating shall be relatively small, and an upper limit for $w / D$ can be defined.

In addition to this, further practical limitations for $\mathrm{w}$ are given by the quality of the environment of the laser engraving set-up (dust particles), and available lasers and optics. The range of linewidths from 10 to $50 \mu \mathrm{m}$ in combination with millimetric line-to-line distances $D$ turned out to represent a reasonable compromise being compatible with all theoretical and practical boundary conditions. An interesting alternative to the square patch pattern might be the hexagonal patch pattern due to its advantageous perimeter to area ratio [29]. However, this pattern does not feature uninterrupted straight lines and would therefore imply a longer processing time and an increase in the precision level in high-speed and large-scale production.

The mechanical properties of the windows are designed to resist mechanical shocks and pressure variations. A treatment should not modify the durability of the substrate. At the chosen wavelength, the glass is transparent. Therefore, we consider that with carefully chosen parameters, laser processing will not impact the mechanical properties of the glazing.

To estimate the impact of the laser treatment on the thermal properties of the coating, the spectral reflectance in the infrared region was measured. For a sample with a 2-mm grid pattern, e.g. less than $4 \%$ of ablated coating, the emissivity increased by $3.2 \%$. The impact of this modification on the overall thermal performance was estimated to be small by calculating the $U$ value for the centre of the glazing.

The selected grid pattern with a linewidth of 35-40 microns has a negligible impact on the direct optical transmittance and the visual appearance of the glazing. This is a key parameter for this new structured coating to be used in replacement of a conventional window. As buildings and trains have a long lifetime, the materials used in their construction should withstand at least 25 years. Compare to these time scales, communication technology evolves very rapidly. Repeaters used in trains and buildings consume energy. The typical power consumption of one repeater is in the order of several tens of $\mathrm{kWh}$ per day. In addition to that, they need to be replaced when the frequencies used for telecommunications change. Therefore, a passive solution to improve the propagation of microwaves in buildings is desirable.

\section{Conclusion}

In conclusion, we have been able to demonstrate by experience and simulation that it is possible to achieve the combination of three key properties.

The transmission of microwaves of the structured coating is similar to the one of the uncoated windows. Even at high angles of incidence, the microwave transmission is dominated by the glass substrate. The impact of this patterning on the attenuation was measured for double glazing, thus taking into account both glass panes and common air gap. Furthermore, only a small fraction of the low emissivity coating was removed. Therefore, the impact of this newly developed structured coating on the overall thermal properties of the window will be relatively small. Besides, the pattern is not distinguishable in most common lighting situations; thus, it does not disturb the view through the window.

To the best of our knowledge, it is the first time that experimental evidence for the combination of such properties is reported and that detailed experimental data are compared to numerical simulations. We expect this new approach to reduce the number and energy consumption needed for repeaters in highly glazed buildings and modern trains while preserving the high thermal insulating properties of the windows.

Acknowledgements We gratefully acknowledge the funding institutions that make this research possible: Swisselectric Research SER, Swiss Federal Office of Transport BFV and to Prof. P. Oelhafen for initiating the project on the energy efficiency in public transportation. We are also thankful to our industry partners for providing materials and services: A. Marguerit, L. Houlmann, N. Noirjean, J. Maushart from AGC Verres Industriels Moutier; N. Dury and R. Holtz from Class4Laser, Lyss; C. Isenschmid from the railway company BLS. We also thank P. Loesch for technical support, L. Maierova for photography, B. Smith and S. Taylor for proof-reading.

\section{References}

1. Mobile network reception problems in low energy buildings. Working group report. Liikenne-ja viestintäministeriö, 2013

2. A. Asp, Y. Sydorov, M. Valkama, J. Niemela, Radio signal propagation and attenuation measurements for modern residential buildings, 580-584 (2012). doi:10.1109/GLOCOMW.2012. 6477638

3. M. Philippakis, C. Martel, D. Kemp, M. Clift, S. Massey, S. Appleton, W. Damerell, C. Burton, Application of FSS Structures to Selectively Control the Propagation of Signals Into and Out of Buildings (ERA report 2004-0072) (Antenna systems, Era technology Ltd, Dorset, 2004)

4. D.R. Smith, W.J. Padilla, D.C. Vier, S.C. Nemat-Nasser, S. Schultz, Composite medium with simultaneously negative permeability and permittivity. Phys. Rev. Lett. 84, 4184-4187 (2000)

5. J. Valentine et al., Three-dimensional optical metamaterial with a negative refractive index. Nature 455, 376-379 (2008) 
6. R.A. Shelby, D.R. Smith, S. Schultz, Experimental verification of a negative index of refraction. Science 292, 77-79 (2001)

7. X. Zheng et al., Ultralight, ultrastiff mechanical metamaterials. Science 344, 1373-1377 (2014)

8. J. Bauer, A. Schroer, R. Schwaiger, O. Kraft, Approaching theoretical strength in glassy carbon nanolattices. Nat. Mater. 15, 438-443 (2016)

9. T.K. Wu, Frequency Selective Surface and Grid Array (Wiley, New York, 1995)

10. B. Munk, Finite Antenna Arrays and FSS (IEEE Press; WileyInterscience, New York, 2003)

11. B. Widenberg, J.V. Rodriguez, Design of Energy Saving Windows with High Transmission at $900 \mathrm{MHz}$ and $1800 \mathrm{MHz}$. in Technical Report LUTEDX/(TEAT-7110/1-14/(2002). https://lup.lub.lu.se/ search/publication/702c9b5d-9daf-4f77-b4fc-da015a0fafab

12. G.I. Kiani, L.G. Olsson, A. Karlsson, K.P. Esselle, Transmission of infrared and visible wavelengths through energy-saving glass due to etching of frequency-selective surfaces. IET Microw. Antennas Propag. 4, 955 (2010)

13. P. Lim, N. Nafarizal, M.Z. Sahdan, S.H. Dahlan, Z. Zainal Abidin, M.Y. Ismail, F. Che Seman, M.K. Suaidi, M.F. Johar, Z.M. Rosli, J.M. Juoi, G.I. Kiani, Optimization of transmission lost for energy saving glass with different sheet resistance values. Adv. Mater. Res. 832, 233-236 (2013)

14. I. Ullah, X. Zhao, D. Habibi, G. Kiani, Transmission improvement of UMTS and Wi-Fi signals through energy saving glass using FSS. IEEE, pp. 1-5 (2011)

15. R. Mittra, C.-H. Chan, T. Cwik, Techniques for analyzing frequency selective surfaces-a review. IEEE Proc. 76, 1593-1615 (1988)

16. F. Costa, A. Monorchio, G. Manara, An overview of equivalent circuit modeling techniques of frequency selective surfaces and metasurfaces. ACES J. 29(12), 963 (2014). http://www.acessociety.org/includes/downloadpaper.php?of=ACES_Journal_ December_2014_Paper_3\&nf=14-12-3

17. F. Costa, A. Monorchio, G. Manara, Analysis and design of ultra thin electromagnetic absorbers comprising resistively loaded high impedance surfaces. IEEE Trans. Antennas Propag. 58(5), 1551-1558 (2010)

18. G. I. Kiani, A. Karlsson, 1. Olsson. Glass characterization for designing frequency selective surfaces to improve transmission through energy saving glass windows. CODEN: LUTEDX/ (TEAT-7170)/1-7/(2008)

19. R. Witte, et al., Investigation of a reliable all-laser scribing process in thin film $\mathrm{Cu}(\mathrm{In}, \mathrm{Ga})(\mathrm{S}, \mathrm{Se})_{2}$ manufacturing, in Proceedings of SPIE 8607, Laser Applications in Microelectronic and Optoelectronic Manufacturing (LAMOM) XVIII, 86071B (2013). doi: $10.1117 / 12.2003872$

20. NF EN 673. Glass in building, Determination of thermal transmittance (U value) Calculation method, eq. 1-5. 2011. ISSN 0335-39. AFNOR

21. H. Rahman, P. K. Saha, J. Dowling, T. Curran, Shielding effectiveness measurement techniques for various materials used for EMI shielding, in IEE Colloquium on Screening of Connectors, Cables and Enclosures, pp. 9/1-9/6 (1992)

22. P.F. Wilson, M.T. Ma, J.W. Adams, Techniques for measuring the electromagnetic shielding effectiveness of materials. I. Farfield source simulation. IEEE Trans. Electromagn. Compatibil. 30(3), 239-250 (1988). doi:10.1109/15.3302

23. C. A. Grosvenor, D. Novotny, R. Johnk, N. Canales, J. Veneman, Shielding effectiveness measurements using the direct illumination technique, in IEEE International Symposium on Electromagnetic Compatibility, 2002. EMC 2002, vol. 1, pp. 389-394 (2002). doi: 10.1109/ISEMC.2002.1032510

24. Agilent "Time Domain Analysis Using a Network Analyzer", Application Note 1287-12, Literature Number 5989-5723EN

25. IEEE Recommended Practice for Measurement of Shielding Effectiveness of High-Performance Shielding Enclosures, in IEEE Std 299-1969, pp. 0-1 (1969). doi: 10.1109/IEEESTD. 1969.120578

26. M. Unser, T. Blu, Generalized smoothing splines and the optimal discretization of the Wiener filter. IEEE Trans. Signal Process. 53(6), 2146-2159 (2005). doi:10.1109/TSP.2005.847821

27. W. C. Gibson, The Method of Moments in Electromagnetics. Boca Raton: Chapman \& Hall/CRC Press, pp. xv + 272 (2008). ISBN: 978-1-4200-6145-1, MR 2503144, Zbl 1175.78002

28. K.A. Michalski, J.R. Mosig, Multilayered media Green's functions in integral equation formulations. IEEE Trans. Antennas Propag 45(3), 508-519 (1997). doi:10.1109/8.558666

29. T.C. Hales, The honeycomb conjecture. Discrete Comput. Geom. 25, 1-22 (2001) 